\title{
THE INFLUENCE OF THE PACKET SIZE ON END TO END DELAY OF VIDEO DATA CODED WITH RAPTORQ CODES AND NETWORK CODES IN VEHICULAR ADHOC NETWORKS
}

\author{
Nandhini Vineeth ${ }^{1}$ and H.S. Guruprasad ${ }^{2}$ \\ ${ }^{I}$ Department of Computer Science and Engineering, BMS College of Engineering, India \\ ${ }^{2}$ Department of Information Science and Engineering, BMS College of Engineering, India
}

\begin{abstract}
The transmission of video files in Vehicular Adhoc Networks (VANETs) has become very prevalent as commuters prefer video data during travel. The delay with which the data is received becomes very significant as video packets received after their scheduled deadlines become useless. The performance of the network may significantly be reduced on such packet drops especially with mobile networks. This work aims at the reduction of end to end delay of video packets by applying the two techniques- Network Coding (NC) and RaptorQ (RQ) codes. The techniques are implemented in four VANET scenarios and an extensive analysis is done by varying the packet sizes during the transmission of three files of various sizes. The End to End Delay (EED) and Packet Delivery Ratio (PDR) are measured and plotted for all scenarios. The results show the influence of packet size on these parameters considered and the suitability of the techniques applied. The observations also show that $R Q$ proves better for smaller files and $N C$ suits better when the file size increases.
\end{abstract}

Keywords:

Fountain Codes, Network Coding, RaptorQ, Vehicular Adhoc Networks, Performance

\section{INTRODUCTION}

Intelligent Transportation Systems (ITS) introduces intelligence in vehicles through the usage of communication among vehicles in VANETs. Vehicular Adhoc Networks, a special stream of Mobile Adhoc Networks are the self-organizing networks formed by the vehicles themselves to communicate with each other. Vehicles communicate among themselves for two main applications.

1. User applications, also termed as infotainment services are generally involved in delivering information to the drivers or passengers like information about the nearby hotels, restaurants, recreation centres etc., and also help passengers be engaged and entertained with music or movie downloads while on travel.

2. Safety applications deal with the transmission of data carrying information on road accidents, traffic jams, bridge fall etc. When such information reaches the vehicles approaching these areas, drivers can make better decisions with the further routes to be taken.

When data is to be transmitted between two vehicles, the distance between the vehicles, the state of the vehicle-mobile or stationary, the speed with which each vehicle move are some of the important parameters that need to be considered. Hence such transmissions from one vehicle to the other become very challenging. Every vehicle which is a part of a VANET is equipped with an On-Board Unit (OBU). The communication links between the OBUs are frequently prone to disconnections as every vehicle has its own speed, direction, random halts etc. The OBUs can also communicate with Road Side Units (RSUs) when long distance communication is required and this is termed as Vehicle to Infrastructure (V2X) whereas the one between two vehicles is termed as Vehicle to Vehicle Communication (V2V).

The data being transmitted may be in any of the forms like text, image, audio, video etc. Among these, video gives a clear visualization of the information to be conveyed but is very challenging. As the vehicles on roads are on move most of the time, transmission of data is a challenging task as frequent link disconnections are experienced. The various parameters of interest in data transmission are throughput, delay, packet loss, etc. When video data is to be transmitted, the end to end delay can be considered as an important parameter as the frames arriving late become useless and are dropped. This results in reduction of the overall performance of the network as many of the other nodes in the network and links help in such data transmission.

This paper is written with the objective of reducing the end to end delay incurred for the transmission of the video data. Two techniques-Network coding (NC) and RaptorQ (RQ) are chosen for working towards the objective. Network coding is chosen as it works towards reduction of delay where simple XOR operation is applied and the number of transmissions is reduced. The prevalent type of NC-Random Linear Network Coding (RLNC) is used in the work. Considering two nodes $A$ and $B$ which are not in the range of each other wants to communicate, they need the support of a relay node. When $A$ wants to transmit a packet say $P$ to $B$ and $B$ wants to transmit a packet say $Q$ to $A$, a relay node $R$ is involved with XORing both packets and the resultant packet $(P \oplus Q)$ is broadcasted. Node A recovers $\mathrm{Q}$ by XORing packet $\mathrm{P}$ with this received XORed packet. $B$ recovers $P$ by XORing $\mathrm{Q}$ with the broadcasted combination. RaptorQ is also chosen here for comparison as it is designed with the aim of complete and quick recovery of data in the destination node. In $R Q$, source packets, say ' $k$ '(numbers) along with a small amount of additional packets say ' $x$ ' are transmitted by the sender. When the receiver receives any $k$ packets out of $k+x$ packets transmitted irrespective of the order or the packet number in the sequence, it will be able to recover the original data. These are special types of fountain codes.

The organization of the paper is as under. The existing literature of the various techniques applied here in the implementation is discussed in section 2. Section 3 details the proposed methodology of this work. Section 4 presents the results measured and plotted for various scenarios and varying parameters and a detailed discussion is done on each observation. Section 5 discloses the conclusion obtained from the 
experimentation and section 6 discusses the future enhancements that could be carried out on this experimentation.

\section{LITERATURE SURVEY}

As this work includes VANETs, Network Coding technique, Fountain codes etc., a survey has been done on these topics. The survey on existing literature is presented here in four groups. The first being the review papers on these topics. The second set summarizes the work on encoding and decoding techniques using $\mathrm{NC}$ and RQ. The third group is on the various works done on delay using the various forms of fountain codes and the fourth includes the set of works where delay has been considered as the significant parameter when network coding is applied.

When new streams of study need to be explored, review or survey papers help us to understand the concepts, the prevalent technologies involved in them, current status of research done in the topic of interest and the open problems that are to be explored.

Researchers have made a detailed comparison of broader areas like Sensor Networks, Adhoc Networks, Mesh networks etc., which gives a clear differentiation between them and help new researchers choose their streams. When VANETs are to be explored, the working of $\mathrm{V} 2 \mathrm{~V}$ can be understood by using Dedicated Short Range Communication (DSRC) which includes protocols like $\mathrm{Wi}-\mathrm{Fi}$, Zigbee etc. Such surveys or reviews give elaborate details about the various technologies, ways of message dissemination, simulators that can be used etc. The suitability of routing protocols across such streams is also an interesting and significant topic of study. Routes can be decided based on different criteria like topology, cluster, position, the set of intersections passed through by a vehicle i.e. trajectory information etc [1]-[3].

Network coding is a very significant technique where the XORing operation is done on the data being transmitted and the number of packets to be transmitted is reduced due to this operation. There are various types of network coding - Random Linear Network Coding which is common and standard type used by many researchers, Generation based NC where the blocks are processed based on generations, Inter session $\mathrm{NC}$ in which packets from two or more different sessions are mixed and broadcasted, Intra session NC where packets from the same sessions are mixed up. The intersession network coding is more advantageous as the two nodes communicating receive their required data simultaneously. This can be applied for both unicasting as well as multicasting scenarios. In network coding procedure, the sender chunks the message into blocks which are seen in a matrix format. Galois field is used and an encoding vector is randomly selected which is combined with the message blocks to be transmitted. The encoding vector is sent with the header of each block by the sender towards the receiver. The receiver applies Gaussian elimination procedure and retrieves back the original block [4]. Network coding is also useful in improving the reliability of safety applications of VANET. These can be applied to various environments like single hop and multihop, sender oriented and receiver oriented and evaluations on these environments can also be done through analytical, numerical and simulation methods [5].

RaptorQ being a Forward Error Correction mechanism, the codes here are proved to be efficient error correction codes which support larger source block sizes, gives reliability and better coding efficiency. Considering the number of source packets to be $k$, with the reception of $N$ packets where $N$ is slightly greater than $k$, the file transmitted can be completely retrieved. Encoder generates as many encoding symbols as needed on the fly. Decoder is able to recover the source block from almost any set of encoded symbols. Encoding symbols of a source block consists of source symbols of the source block and repair symbols generated from the source block. The source symbols are used to generate the intermediate symbols using an inverse encoding process. Repair symbols are generated from Intermediate symbols. As any set of the droplets from a fountain can used to fill a container, any set of $k$ packets collected can be used to decode the complete transmitted data. These codes have become the base code to work on for many researchers. Degree distribution technique which is a probability distribution method is used to begin the RQ technique by generating the number of symbols that can be XORed together and such a XORing generates an encoded symbol. Precoding is done in two stages here. The first precoding uses a low density parity check (LDPC) code to generate redundant symbols whereas the second precoding stage uses high density parity check (HDPC) codes. An efficient version of Gaussian elimination is used in the decoder to recover back the source symbols [6].

\subsection{ENCODING AND DECODING}

Researchers have introduced new mechanisms in encoding and decoding with the objective of enhancing the efficiency of wireless networks. A MAC mechanism has been designed which works towards the enhancement in performance of Raptor codes used with Orthogonal Frequency Division Multiplexing systems. The authors give prominence to the CRC being done with actual fountain encoded packets and set an energy threshold. The packets which are received with energy less than a threshold are dropped and the ones with energy above this are considered and error correction is done with the help of CRC codes [7]. New techniques have been introduced by researchers one being the zigzag decodable FC which is a shift and XOR done on the data compared to the simple XOR done with FC which results in a reduced decoding overhead and erasure rate [8]. The other new introduction has been the Micro random code which takes an overhead of one symbol in comparison to ten overhead symbols used by random code. The small message transmission which is very challenging with fountain codes have been done where micro symbols are used with message symbols for encoding [9].

The performance efficiency is improved by approaches which work on efficient encoding, decoding in source or destination and sometimes also in the intermediate stages. An efficient encoding is required in content centric networking to show the achievement of better performance of these networks in terms of hit ratio in cache, download time, amount of data transmitted and hence the authors in [10] have proposed a request specified NC which introduces an efficient encoding in the multiple sources and the improvised network cache. The ideas of forwarding interest, receiving interest, the action taken when a packet arrives at an interface etc., are presented in the form of algorithms. Conversions from a standard format of Real Time Protocol to a user extended format termed as Erasure Coding RTP have also shown good results for bandwidth, packet delay, loss and image 
quality. Such a conversion is tried out in the boundary line of Internet and VANET in [11] which shows a better performance compared to conventional methods. Efficient decoding is achieved in [12] where a multi edge framework scheme based on Belief Propagation decoding in RQ is considered. The joint decoding concept is implemented where the component codes of inner code and precode are decoded in parallel and the information retrieved is shared among them to show better optimization.

The efficiency of the network can also be enhanced by using a cooperative communication among the nodes in the network. A thesis work based on cellular networks with the design is carried out on the cooperative techniques based on RLNC. The mobile cloud is introduced where mobile nodes get together to form a cluster. A unicast scenario with a single source and multiple relays is established and the appropriate number of nodes in each cloud to achieve efficient data rate is shown. An increase in throughput and reduction of energy consumed in nodes as well as the base station are presented as the result [13]. Similarly it is observed that not all nodes would be interested in forwarding the data of others. There are incentive based methods which benefit the nodes forwarding the data towards destination and thus increases the overall successful data transmission in the network [14]. Cooperative communication is also exhibited when vehicles are grouped together and prioritized based on their usage Emergency vehicles (ambulance, fire engine etc.), VIP vehicles and vehicles of common use. Hence the priority is given to packets to and from these in the order as seen above and the end to end delay incurred is reduced. This is achieved with the cooperation from the peer vehicles [15].

Fountain codes and network codes are used predominantly in the reduction of delay. This has been the motivation for the exploration of these techniques in this work.

\subsection{DELAY REDUCTION USING FOUNTAIN CODES}

When multimedia transmission is involved, fountain codes have proven to give better results. Rateless coding are channeladaptive and the video coding are delay aware. These two features are applied in Internet of Things. The combination of the Model Predictive Control technique into the delay aware sliding window based fountain codes has shown to result in an optimum decoding ratio which outperforms many video communication algorithms in [16]. The work is extended in [17] to show that the video quality is significantly improved by adaptively selecting the window size. Many researchers focus on the relay nodes which play a major role in such multimedia transmissions. As delay is a major issue with their usage, the boundary region between the fountain coding-based and classical relay system is found in [18] and the coding-based system is shown to give a lesser transmission delay. The work also shows the influence of the packet size, message size and the signal to noise ratio on delay in relay based systems. A new protocol is proposed in [19] which select the best relay node for multimedia transmissions involving time sharing. With the assumption of the minimum block error rate and the link being two hops, the decoding parameters like overhead, probability of failure is shown to outperform the conventional amplify and forward scheme. When such decoding success probability of random linear FC is focussed, redundancy of received data is high which is shown to be reduced in [20] by making a codeword which is not a linear combination of a predetermined numbers of code words. The number of transmissions is shown to be significantly reduced for multicast networks.

An integration of LT code based application layer channel coding and multicasting in VANETs is applied in [21] which works towards improvising the throughput and end to end delay of the data. Two phases of data dissemination where data gets transmitted from the RSU to the vehicles and also from one vehicle to another is done. The technique gives a better arrival time of packets compared to the conventional techniques. Another such integration of that of the FLUTE-File Delivery over Unidirectional Transport protocol and the RaptorQ codes to multicast video data is done in [22]. The source blocks are made from transport objects created from the trace files. Markov model is used for modelling the losses. The evaluation of Peak Signal to Noise Ratio, startup delay and the percentage of re-buffering are done and a better decoding strategy is shown.

\subsection{DELAY REDUCTION USING NETWORK CODES}

Network coding has also proved to work well with delay. Delay Analysis is done in with various ways based on routing protocols, mobility models, link quality etc. Different routing protocols like Open Link State Routing Protocol (OLSR), Destination Sequenced Distance Vector (DSDV) and Adhoc on Demand Routing Protocol (AODV) are applied in [23], a buffer is used in combination with an erasure code in [24], various Mobility Models (MM) like Constant Position MM which is exhibited by stationary vehicles, Constant Velocity MM where all vehicles move with the same velocity, Constant Acceleration MM where all vehicles move with the same acceleration in [25]. It is also analysed in [26] that when inter-session NC is applied for two unicast sessions with hard deadlines, low performance is observed when the file sizes are small or medium. When an Immediately or Instantly decodable Network Coding is used, the performance of file transmission for small files is improved. An analysis is done in [27] based on the link quality between the vehicles where a backbone of vehicles is formed dynamically which broadcast packets when multihop disseminations are involved and the packet delivery is shown to have improved.

Researchers have also used the dynamic programming in proving optimality of their system in terms of file transfer completion time. The system in [28] has been modelled as a Markov decision process and oriented towards the storage and the computational complexity in receiver though RLNC. The process gives a good throughput and less transmission delay in broadcast network. Reliable delivery of data is the objective in [29]. Data transmission is done in two phases- first phase being uncoded and the second where the packets belonging to the same generation are grouped and encoded. Their new requirement is that the intermediate node in RLNC need to provide feedback regarding the status of packets received in each receiver node after the first phase. They work on throughput, decoding delay of the packets. Generations are further divided into sub generations and every packet transmitted is a linear combination of packets that belong to the same sub-generation. This is shown as a NP Complete problem and an algorithm is presented as a solution which gives better results compared to other solutions. An NC scheme with 
cooperative data exchange is used which could help in maximizing the number of packets received within their deadlines. These can be applied to sessions with frequent data exchanges. The problem is proved NP-hard in [30] by formulating into integer programming. Auxiliary graph model is used by authors to design an algorithm which could help in solving the general problem.

Another work on prioritization in [31] applies network coding during video transmission in multicast scenarios with mobile nodes. The packets here are prioritized and the focus is on reducing the drops and transmission errors of high priority packets. A previous work of the authors is Multicast Scalable Video Transmission using Classification-Scheduling Algorithms and Network Coding over MANET (MSVT_CSA_NC) [32]. Here this technique is an extension named Extended MSVT_CSA_NC. In both these work, a cross layer design is involved. The difference between the two lies with the source and the intermediate node processing. A classification algorithm is used in the earlier work to adjust the block size and the intermediate node makes blocks according to packet priorities. The current work makes dynamic block sizes based on inter layer compensation and then packets are encoded. This evaluates and shows improved delay, throughput and packet delivery ratio. They vary the network coding technique by making all the blocks of the packets to same size.

\section{PROPOSED METHODOLOGY}

The two techniques- Network Coding and RaptorQ codes as seen in the above literature are used very predominantly to reduce the end to end delay in wireless networks in general. Hence the performance analysis of these two techniques becomes essential to check the suitability of either of them to a given scenario. This motivation has resulted in this work where the performance of both these techniques are analysed by applying them to the same environments. The significant parameters End to End Delay (EED) and the Packet Delivery Ratio (PDR) are measured.

The communication between the vehicles are varied by modifying the scenario with various possible combinations of single or two hops with single or two transmissions.

1. Single Hop Single Transmission (SHST) where two nodes which are in range of each other communicate and it is assumed that no other transmission is seen in the network.

2. Multiple Hops Single Transmission (MHST) where nodes that are not in the range of each other wish to communicate. The number of hops between the communicating nodes is two or more. It is assumed that no other transmission is seen in the network.

3. Single Hop Multiple Transmissions (SHMT) where multiple pairs of nodes which are in the range of the each other communicate. The number of transmissions encountered here are two or more. In this work, two pairs of vehicles are involved in transmission.

4. Multiple Hop Multiple Transmissions (MHMT) where multiple pairs of nodes which are at a minimum distance of two hops with their pair node communicate. The number of transmissions encountered here are two.
The sequence of steps followed during implementation is listed below.

\subsection{ALGORITHM FOR THE IMPLEMENTATION PROCEDURE}

1. A grid topology of twenty VANET nodes is set up.

2. The routing protocol-AODV is installed and the mobility model where the vehicles move with different but constant velocities is set up.

3. VANET environment is set up by implementing $802.11 \mathrm{p}$ WAVE standards with packet size as 1024 bytes.

4. The simplest scenario Single Hop Single Transmission is implemented.

5. RaptorQ encoder module that uses LDPC and HDPC codes in the precoding stage is supplied the video data which encodes the data and the encoded packets are transmitted towards the receiver which decodes them using belief-propagation Gaussian elimination.

6. The Network coding module uses the random coefficient vector chosen from the Galois field applied on the data block to encode the packets. The receiver receives the packets and check for innovative and non-innovative packets. Innovative packets are the ones that give a new packet to the receiver which is included in the decoding matrix. Non innovative packets add a row with zeros in the decoding matrix. Gaussian elimination technique which involves converting the received matrix into an upper triangular matrix followed by back substitution is used to extract the original block from this matrix. This module is applied to the setup and the experiment is repeated.

7. Three video files of different sizes are transmitted in each of these techniques. The flow in the network is monitored.

8. The packet sizes are varied as $64,128,256,512$ and 1024 bytes and the EED and PDR are measured for each scenario when different files with sizes $10 \mathrm{~KB}, 20 \mathrm{~KB}$ and $38 \mathrm{~KB}$ are transmitted.

9. The same nodes are maintained as the client and server nodes in all the three techniques to maintain identical observations. When the technique is Network coding, a node which is one hop from the client and one hop from the server is chosen as the relay node which does the recoding of packets and the EED and PDR are measured and plotted.

10. The steps 5 to 8 are repeated for all the other three scenarios.

\section{RESULTS AND DISCUSSION}

The communication among twenty vehicles is considered in this work. This is scalable to any numbers. When the number of vehicles is increased, it becomes practically difficult to set up realtime VANET testbeds. Hence Network Simulator 3 is used for implementing the algorithm discussed above. The various criteria considered for implementation are tabulated below in Table.1. 
Table.1. Various Criteria considered for implementation

\begin{tabular}{|c|c|}
\hline Criteria & Value \\
\hline No. of Vehicles & 20 \\
\hline Mobility model & $\begin{array}{c}\text { Constant Velocity Mobility } \\
\text { model with vehicles moving } \\
\text { with different but constant } \\
\text { velocities }\end{array}$ \\
\hline Speed of Vehicles & $\begin{array}{c}1 \text { to } 10: 40 \mathrm{~km} / \mathrm{hr} ; 11 \text { to } 20: \\
80 \mathrm{~km} / \mathrm{hr}\end{array}$ \\
\hline Multiplexing & OFDM \\
\hline Data rate & $12 \mathrm{Mbps}$ \\
\hline Bandwidth & $10 \mathrm{MHz}$ \\
\hline Routing Protocol & AODV \\
\hline Transmitted file size & $10 \mathrm{~KB}, 20 \mathrm{~KB}, 38 \mathrm{~KB}$ \\
\hline
\end{tabular}

AODV protocol is chosen in this work as this is a reactive protocol which makes routing decisions dynamically. As the movement of vehicles takes place with varying velocities, the links between the vehicles which help in transmission of data may be cut very frequently. Hence the reactive algorithm AODV best suits the environment. The WAVE standards are implemented using the modules implementing 802.11p. Packet sizes are chosen to adapt to the underlying channel capacity available. The two parameters that are considered as input parameters in this implementation are the file size and the packet size. Three files of varying sizes as $10 \mathrm{~KB}, 20 \mathrm{~KB}$ and $38 \mathrm{~KB}$ are considered for transmission. The difference here is that the packet size is varied as $64,128,256,512$ and 1024 bytes during the transmission of all the three files. The EED and the PDR in each transmission is measured. Results of the monitored details are plotted as graphs. The PDR values mentioned in all graphs represent the Packet Delivery Ratio measured when RQ is applied. As 100\% PDR was observed in all cases when $\mathrm{NC}$ is applied, no mention of the same is done in the graphs.

\subsection{FILE SIZE: 10KB}

The EED measured when a file with size $10 \mathrm{~KB}$ is transmitted in the various scenarios are plotted and shown below.

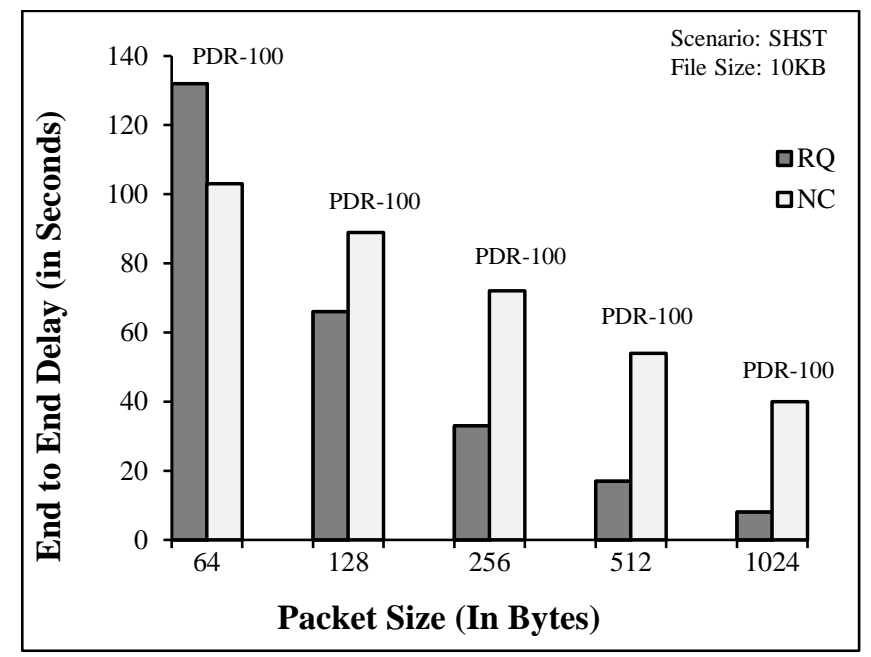

Fig.1. EED Observed in SHST with File Size 10KB

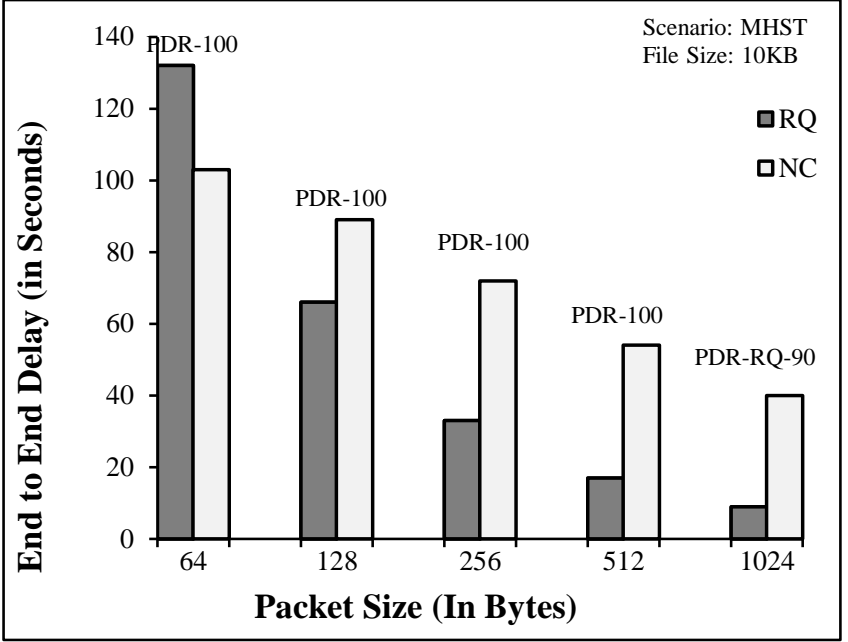

Fig.2. EED Observed in MHST with File Size 10KB

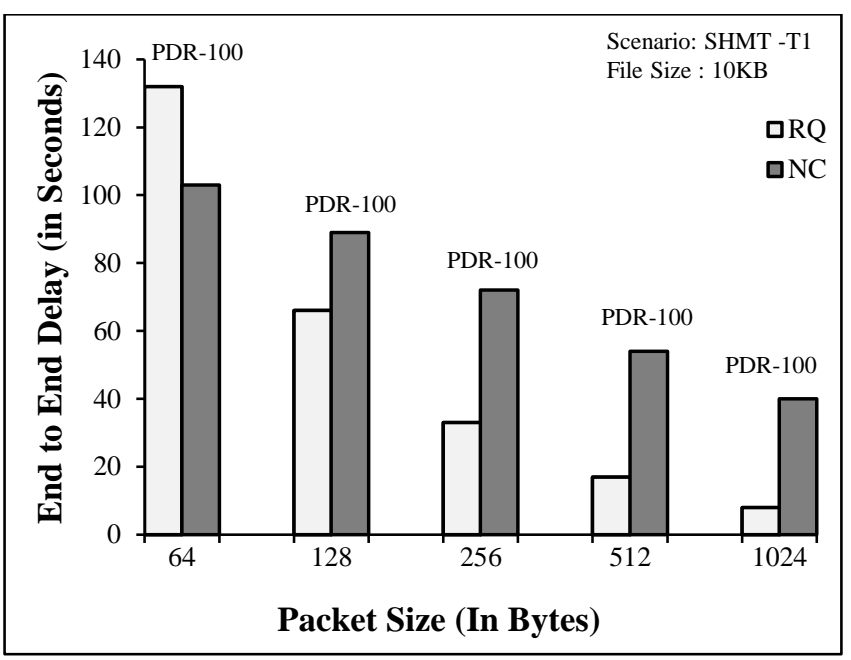

Fig.3. EED Observed in SHST-T1 with File Size 10KB



Fig.4. EED Observed in SHST-T2 with File Size 10KB 




Fig.5. EED Observed in MHMT-T1 with File Size 10KB

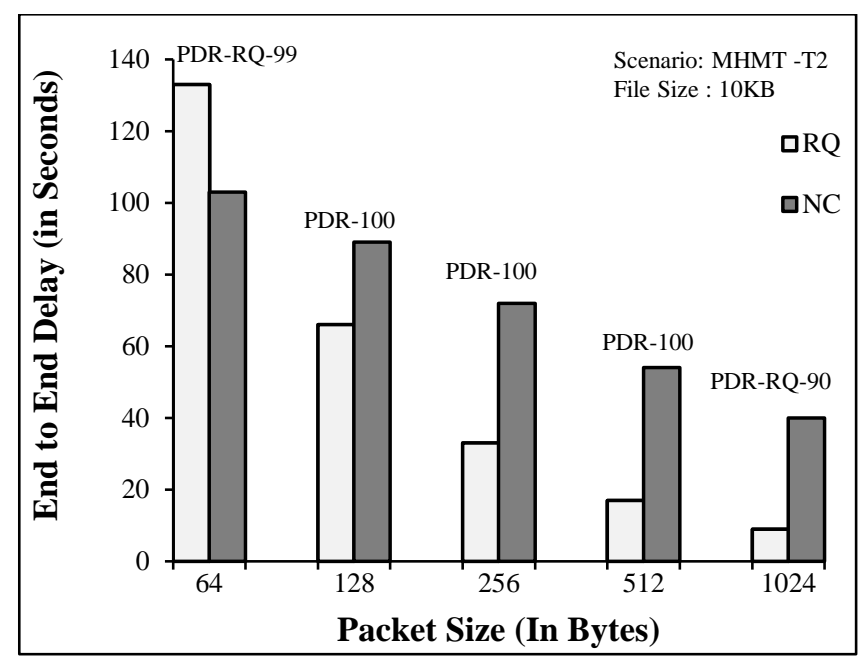

Fig.6. EED Observed in MHMT-T2 with File Size 10KB

\subsubsection{Observations of EED:}

- From the Fig.1 to Fig.6 with regard to EED, when the packet size is 64 bytes, $\mathrm{NC}$ achieves a lesser delay than RQ in all scenarios set. With all other packet sizes, RQ outperforms NC.

- With regard to the packet drops, a significant drop is seen with PDR being 50\% only for RQ in MHMT-T1 Scenario. This is the result of two simultaneous transmissions, each with two hops in the network and also the usage of least packet size.

- The NC packets show $100 \%$ delivery in all cases and hence no mention is done in the graph.

\subsection{FILE SIZE: 20KB}

The EED measured when a file with size $20 \mathrm{~KB}$ is transmitted in the various scenarios are plotted and shown as follows.



Fig.7. EED Observed in SHST with File Size 20KB

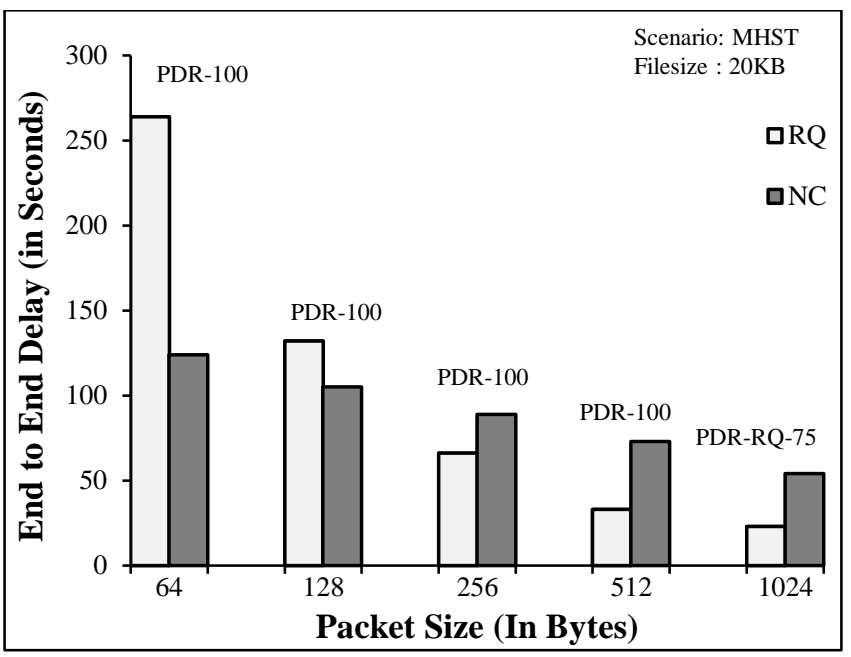

Fig.8. EED Observed in MHST with File Size 20KB



Fig.9. EED Observed in SHMT-T1 with File Size 20KB 




Fig.10. EED Observed in SHMT-T2 with File Size 20KB

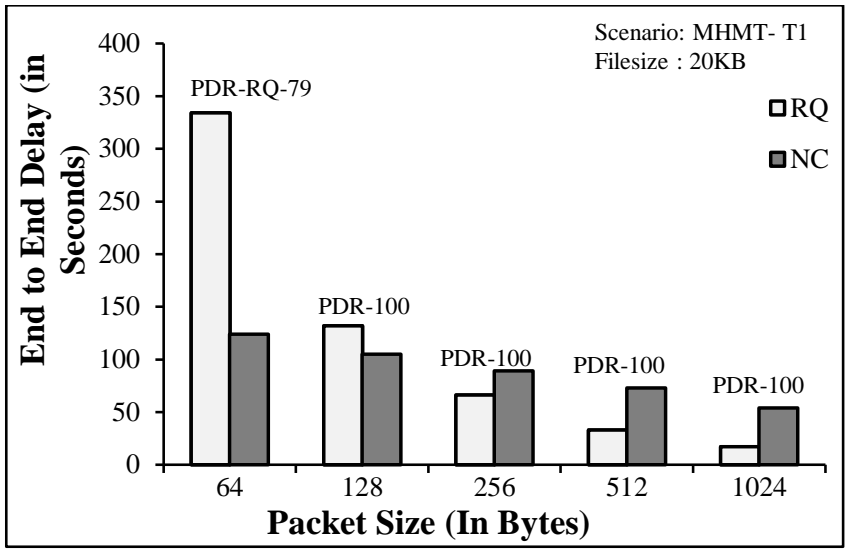

Fig.11. EED Observed in MHMT-T1 with File Size 20KB



Fig.12. EED Observed in MHMT-T2 with File Size 20KB

\subsubsection{Observations of EED:}

- With regard to EED (Fig.7 to Fig12), when the packet size is set to 64 and 128, NC gives a better end to end delay compared to RQ

- With regard to EED, when the packet size is set to 256, 512 and 1024, RQ gives a lesser delay when compared to $\mathrm{NC}$

- As the file size increases, the observation is that the packet drops increases in MHST scenario where packets travel with two hops.
- When multiple transmissions are done, one transmission is done efficiently whereas the other experiences packet drops. This is the result of two simultaneous transmissions, each with two hops in the network and also the usage of least packet size.

- The NC packets show $100 \%$ delivery in all cases and hence no mention is done in the graph.

\subsection{FILE SIZE: 38KB}

The EED measured when a file with size $20 \mathrm{~KB}$ is transmitted in the various scenarios are plotted and shown below.



Fig.13. EED Observed in SHST with File Size 38KB

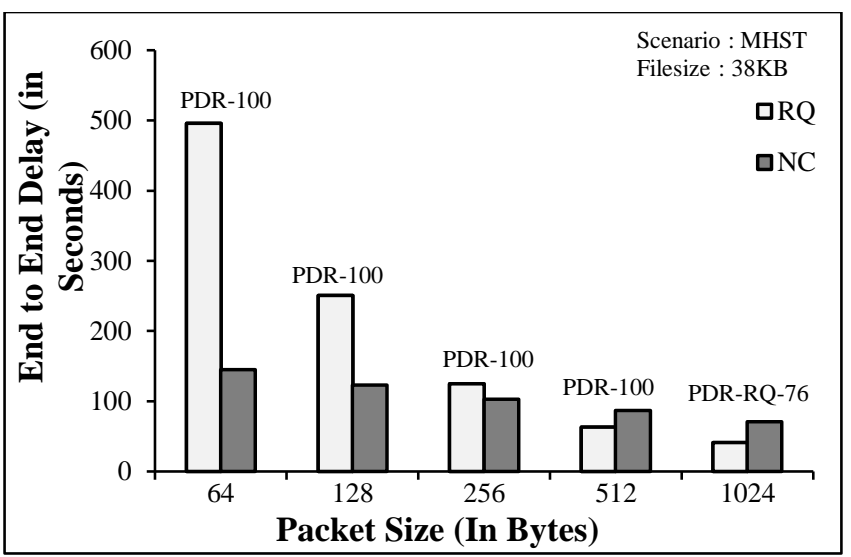

Fig.14. EED Observed in MHST with File Size 38KB

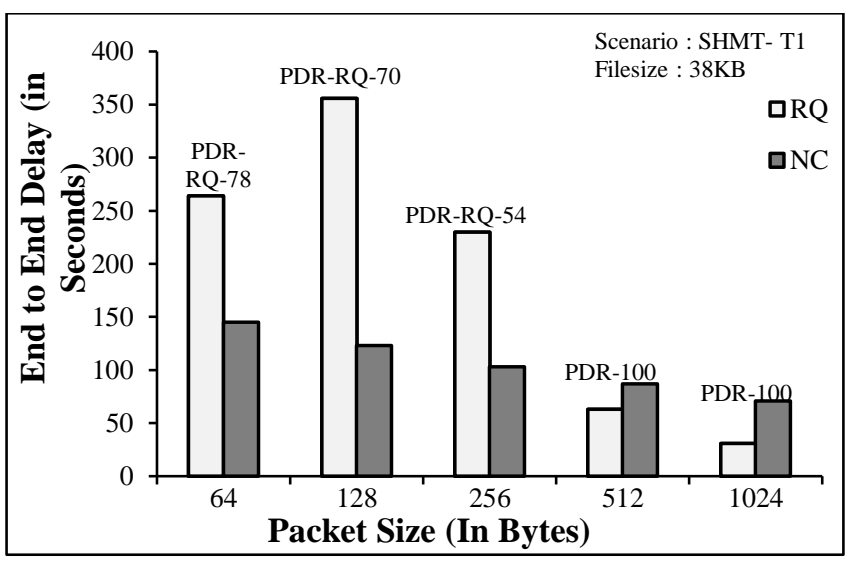

Fig.15. EED Observed in SHMT-T1 with File Size 38KB 


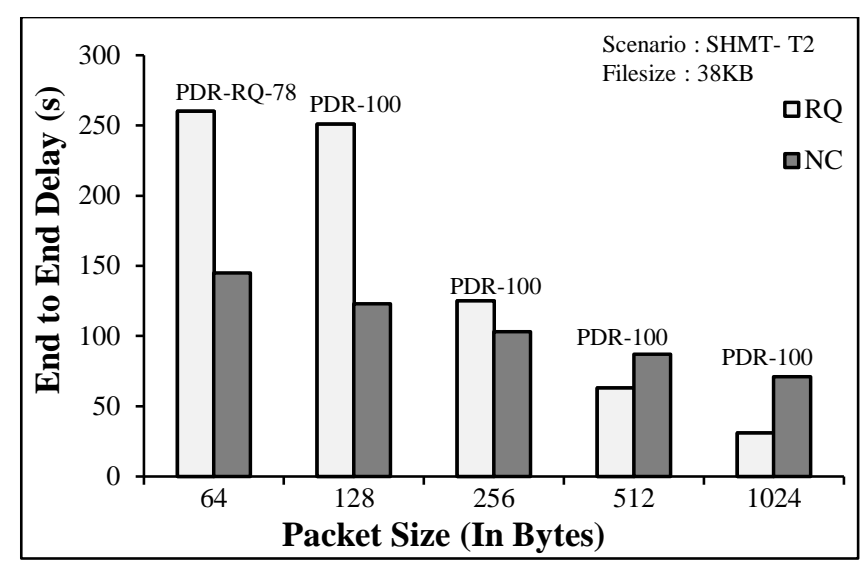

Fig.16. EED Observed in SHMT-T2 with File Size 38KB

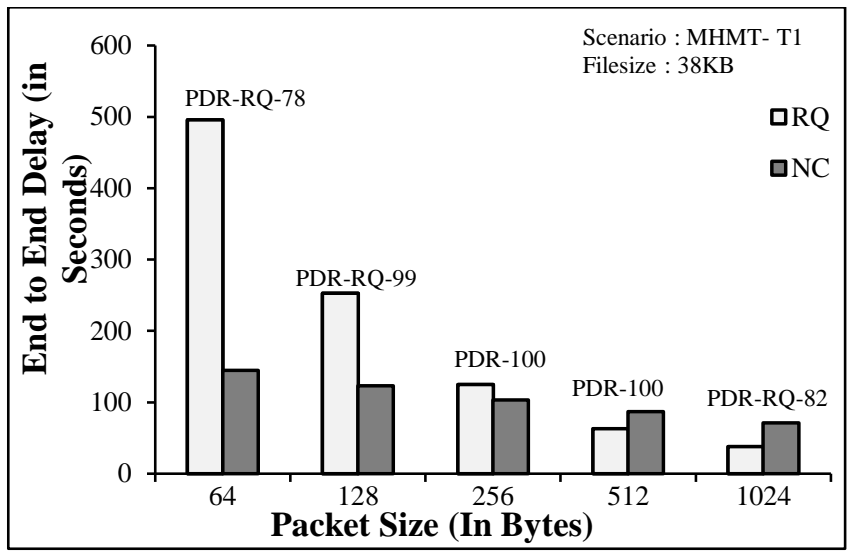

Fig.17. EED Observed in MHMT-T1 with File Size 38KB

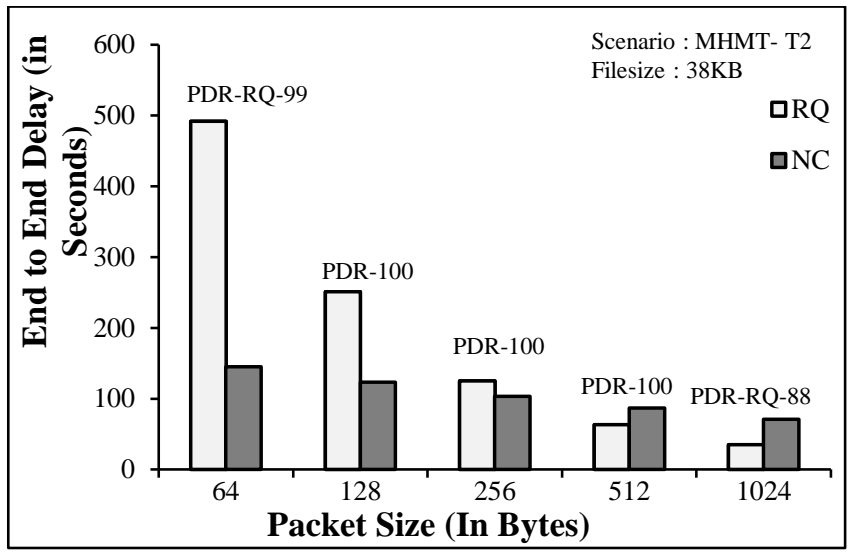

Fig.18. EED Observed in MHMT-T2 with File Size 38KB

\subsubsection{Observations of EED:}

- With regard to EED (Fig.13-Fig.18), when the packet size is set to 64,128 and 256, NC gives a better EED compared to $\mathrm{RQ}$

- With regard to EED, when the packet size is set to 512 and 1024, RQ gives a lesser EED when compared to NC

- When the file size is increased further, we are able to observe the packet drops in both transmissions when multiple transmissions are done.
- The NC packets show $100 \%$ delivery in all cases and hence no mention is done in the graph.

A general observation here is that both NC and RQ exhibit a reduction of EED with an increase in packet size. The reason for this is that when the packet size is small, the headers become predominant and the data carried becomes less in every packet. The number of packets increases due to less packet sizes. The processing of headers of each packet takes up more time and hence results in an increase of the overall end to end delay.

Another observation that can be made is that, the packet drops are more in the multi hop scenarios when RQ is applied. This infers that with increase in distance RQ transmission drops more packets. RQ being a FEC mechanism and a technique concerned with erasure channels like that used by VANETs, it is taken care that additional repair symbols are produced along with source symbols by the encoder and both are transmitted and hence this is not a very serious constraint as it looks. As seen in the literature, as soon as the number of symbols arriving at the destination reaches the number of source symbols irrespective of whether it is a source or repair symbol, the decoding can be done. As $10 \%$ additional symbols are used in the experimentation, when the PDR is $90 \%$ and above, the packet drops can be ignored but the ones measured less than $90 \%$ needs a significant attention. In NC, a better packet delivery is exhibited in multihop scenario for the significant reason that a relay node in the intermediate stage involving in recoding and transmission of the packets.

Summarizing all the above graphs, as the file size increases, NC exhibits a better performance compared to RQ with smaller packets. We are able to infer that when a huge file needs to be transmitted, NC can be used for transmission and RQ is suitable for smaller files with respect to end to end delay. With NC and RQ, the least EED is observed, when every packet is transmitted with a size of 1024 bytes proving the significance of the packet size.

\section{CONCLUSION}

When video data needs to be transmitted in VANET scenarios, the end to end delay and the packet delivery ratio are very important parameters to be considered and hence are focussed in this work. The Network Coding technique and the RaptorQ technique which are proven to show a good performance in wireless networks are considered for comparison. As a result of the analysis done, the size of the packet is shown to have a strong influence on the end to end delay. The results obtained help us conclude that when the file size is smaller, RaptorQ codes can be considered as the technique compared to NC as it exhibits an improved performance with increase in the packet size. When a larger file is transmitted, Network coding technique is proven to be a better choice as it shows an improvement over the RaptorQ codes with increasing packet sizes. Network coding technique and RaptorQ codes exhibit a better performance with packet size being 1024 bytes compared to the lesser sizes.

\section{FUTURE ENHANCEMENT}

When RQ is applied, as the PDR is observed to be reduced with increasing distance, a strategy needs to be worked on in future for the same. As both these techniques NC and RQ exhibit their own significance with different environments, the future 
enhancement of this work is the design of an adaptive technique which dynamically chooses and applies either NC and RQ appropriately which adapts to the prevailing environment and could outperform the other.

\section{REFERENCES}

[1] Elias C. Eze, Si-Jing Zhang, En-Jie Liu, Joy C. Eze, "Advances in Vehicular Ad-Hoc Networks (VANETs): Challenges and Road-Map for Future Development", International Journal of Automation and Computing, Vol. 13, No. 1, pp. 1-18, 2016.

[2] Erika Rosas et al., "Survey on Simulation for Mobile AdHoc Communication for Disaster Scenarios", Journal of Computer Science and Technology, Vol. 31, No. 2, pp. 326349, 2016.

[3] Hafi Houda and Merniz Salah, "A Survey of Trajectory based Data Forwarding schemes for Vehicular Ad-Hoc Networks", Proceedings of IEEE International Conference on Communication Software and Networks, pp. 399-404, 2015.

[4] Nandhini Vineeth and H.S. Guruprasad, "The Influence of Network Coding on The Performance of Wireless Networks: A Survey", International Journal of Advanced Computer Technology, Vol. 3, No. 6, pp. 884-892, 2014.

[5] Shehu Jabaka Muhammad, Sijing Zhang and Vladimir Dyo, "Network Coding for Reliable Safety Message Communication in Vehicular Ad-Hoc Networks: A Review", Proceedings of IEEE $4^{\text {th }}$ International Conference on Future Generation Communication Technology, pp. 125131, 2015.

[6] David J.C. MacKay, "Fountain Codes", IEEE Proceedings Communications, Vol. 152, No. 6, pp. 1062-1068, 2005.

[7] N.M. El-Gohary, Mohsen A.M. Kassem, F.E. Abed ElSamie and M.M. Fouada, "Performance Enhancement of Raptor Codes Employment in OFDM-System", International Journal of Networks and Communications, Vol. 6, No. 6, pp. 103-110, 2016

[8] Takayuki Nozaki, "Zigzag Decodable Fountain Codes", IEICE Transactions on Fundamentals of Electronics, Communications and Computer Sciences, Vol. 100, No. 8, pp. 1693-1704, 2016

[9] Chong Zan Kai, "Rateless Erasure Codes for Short Messages Transmission”, Ph.D Dissertation, Department of Engineering and Science, Universiti Tunku Abdul Rahman, 2016.

[10] Yan Liu and Shun-Zheng Yu, "Network Coding-based Multisource Content Delivery in Content Centric Networking", Journal of Network and Computer Applications, Vol. 64, pp. 167-175, 2016.

[11] Abdelhamid Mammeri, Azzedine Boukerche and Zhifei Fang, "Video Streaming over Vehicular Ad Hoc Networks using Erasure Coding”, IEEE Systems Journal, Vol. 10, No. 2, pp. 785-796, 2016.

[12] Sachini Jayasooriya, Mahyar Shirvanimoghaddam, Lawrence Ong and Sarah J Johnson, "Analysis and Design of Raptor Codes using a Multi-Edge Framework", Information Technology, Vol. 10, No. 4, pp. 1-30, 2017.
[13] Nestor Javier Hernandez Marcano, "Network Coding for Cooperation in Wireless Networks", Ph.D. Dissertation, Aalborg University, pp. 1-106, 2016.

[14] Brij Bihari Dubey, Naveen Chauhan, Narottam Chand and Lalit Kumar Awasthi, "Incentive based Scheme for Improving Data Availability in Vehicular Ad-Hoc Networks", Wireless Networks, Vol. 23, No. 6, pp. 1-19, 2016.

[15] Alfred Daniel, Anand Paul, Awais Ahmad and Seungmin Rho, "Cooperative Intelligence of Vehicles for Intelligent Transportation Systems (ITS)", Wireless Personal Communications, Vol. 87, No. 2, pp. 461-484, 2016.

[16] Kairan Sun, Huazi Zhang, Dapeng Wu and Hongcheng Zhuang, "MPC-based Delay-Aware Fountain Codes for Real-Time Video Communication", Proceedings of IEEE International Conference on Communications, pp. 1-2, 2016.

[17] Kairan Sun, Huazi Zhang and Dapeng Wu, "Delay-Aware Fountain Codes for Video Streaming with Optimal Sampling Strategy", IEEE Multimedia, Vol. 15, No. 3, pp. 1-12, 2016.

[18] Arash Asareh and Takeo Fujii, "Delay Reduction Scheme based on Fountain Coding for Wireless Relay Communication Systems", Proceedings of $22^{\text {nd }}$ Asia-Pacific Conference on Vehicular Technology, pp. 1-6, 2014,

[19] Muhammad Talha Gul, Amjad Ali, Deepak Kumar Singh , Umera Imtinan , Imran Raza, Syed Asad Hussain ,Doug Young Suh and Jong-Wook Lee, "Merge-and-Forward: A Cooperative Multimedia Transmissions protocol using RaptorQ Codes", IET Communications, Vol. 10, No. 15, pp. 1884-1895, 2016.

[20] Jalaluddin Qureshi, "Random Linear Fountain Code with Improved Decoding Success Probability", Proceedings of $22^{\text {nd }}$ Asia-Pacific Conference on Networking and Internet Architecture, pp. 1-6, 2017.

[21] Veronica Palma, Elena Mammi and Anna Maria Vegni, “A fountain Codes-based Data Dissemination Technique in Vehicular Ad-Hoc Networks", Proceedings of $11^{\text {th }}$ International Conference on ITS Telecommunications, pp. 750-755, 2011.

[22] Utsaw Kumar and Ozgur Oyman, "QoE Evaluation for Video Streaming over eMBMS", Proceedings of IEEE International Conference on Computing, Networking and Communications, pp. 555-559, May 2013.

[23] Nandhini Vineeth and H.S. Guruprasad, "Delay Analysis of Network Coded Video Streams in VANETs", International Journal of Information Engineering and Electronic Business, Vol. 4, pp. 16-23, 2015.

[24] Jesper H Sorensen, Petar Popovski and Jan Ostergaard, "Delay Minimization in Real-time Communications with Joint Buffering and Coding", IEEE Communications Letters, Vol. 21, No. 1, pp. 52-55, 2017.

[25] Nandhini Vineeth and H.S. Guruprasad, "Performance Analysis of Network Coded Video Streams in VANETs based on Mobility Models", Proceedings of IEEE International Advance Computing Conference, pp. 170-175, 2015.

[26] Xiaohang Li, Chih-Chun Wang and Xiaojun Lin, "InterSession Network Coding Schemes for 1-to-2 Downlink Access-Point Networks With Sequential Hard Deadline 
Constraints", IEEE/ACM Transactions on Networking, Vol. 25, No. 1, pp. 624-638, 2017.

[27] Celimuge Wu, Xianfu Chen, Yusheng Ji, Satoshi Ohzahata and Toshihiko Kato, "Efficient Broadcasting in VANETs using Dynamic Backbone and Network Coding", IEEE Transactions on Wireless Communications, Vol. 14, No. 11, pp. 6057-6071, 2015.

[28] Emmanouil Skevakis and Ioannis Lambadaris, "Optimal Control for Network Coding Broadcast", Proceedings of Global Communications Conference, pp. 1-6, 2016

[29] Mingchao Yu, Parastoo Sadeghi and Alex Sprintson, "Feedback-Assisted Random Linear Network Coding in Wireless Broadcast", Proceedings of IEEE Globecom Workshops, pp. 1-6, 2017.
[30] Yang Sui, Xiumin Wang, Jin Wang, Lusheng Wang and Saihang Hou, "Deadline-Aware Cooperative Data Exchange with Network Coding", Computer Networks, Vol. 97, pp. 88-97, 2016.

[31] Olfa Ben Rhaiem, Lamia Chaari Fourati and Wessam Ajib, "QoS Improvement for Video Streaming over MANET using Network-Coding", Proceedings of IEEE $82^{\text {nd }}$ Vehicular Technology Conference, pp. 1-5, 2015.

[32] Olfa Ben Rhaiem, Lamia Chaari Fourati, Wessam Ajib, "Network Coding-based Approach for Efficient Video Streaming over MANET", Computer Networks, Vol. 103, pp. 84-100, 2016. 